\title{
Sink depth of trolled fishing lines
}

\section{G. Spolek}

Mechanical \& Materials Engineering, Portland State University, Portland, USA

\begin{abstract}
During recreational troll fishing, success usually depends on the ability to maintain the lure at the desired depth. When trolling with a heavy line, one whose inherent density sinks the lure, depth control is guesswork for most anglers. This study presents a method for predicting the trolling depth of heavy fishing lines. The model is developed based on the fluid mechanics of the line being towed through quiescent water and determines line depth for known line properties and trolling speed. Experiments are performed to validate the model and results are consistent with the model predictions. The model is employed to illustrate how trolling depth can be controlled during typical fishing situations.
\end{abstract}

Keywords: fishing, line depth, trolling

\section{Introduction}

Of the many recreational fishing methods commonly used, lake trolling (harling) is one of the most popular. In trolling, a boat is used to pull a lure through the water at the depth of the fish. The lure simulates a fish's food item, such as a minnow or insect, through its size, colour, and swimming motion. Since fish tend to suspend at a specific depth within the water column, trolling success depends upon being able to keep the lure at the same depth as the fish.

Sinking a lure to the desired depth can be accomplished many ways. The lure may be heavy enough to sink or a lead sinker can be attached to the line that pulls the lure downward. Another method employs a

Correspondence address:

Graig Spolek

Mechanical \& Materials Engineering

Portland State University

POB 751 Portland, OR 97207, USA

Tel: + 0015037254293

Fax: + 0015037258255

E-mail: graig@cecs.pdx.edu heavy line that sinks its free tip to the desired fishing depth, to which is attached a very lightweight wobbling lure or fly; this method is commonly used for trout and kokanee. Because these types of fish are depth-sensitive, the odds of success hinge upon obtaining the correct depth for a sinking trolled line.

This paper establishes a simple method to determine the depth of a trolled heavy fishing line.

\section{Background}

While trolling, the angler will seek to maintain constant boat speed to mimic the swimming speed of the food item. Finding the right lure depth at that speed is normally a trial and error process: pay out more or less trolled line, mark the amount of line being used when a fish is hooked, and then repeat. Some fishing lines, such as lead core trolling lines, are marked with different colours to facilitate measuring the amount of line paid out. Many boats will have electronics to accurately measure boat speed (via GPS) and fish depth (via sonar). What is missing, however, is the relationship between the line payout and subsequent lure depth for a given boat speed. 


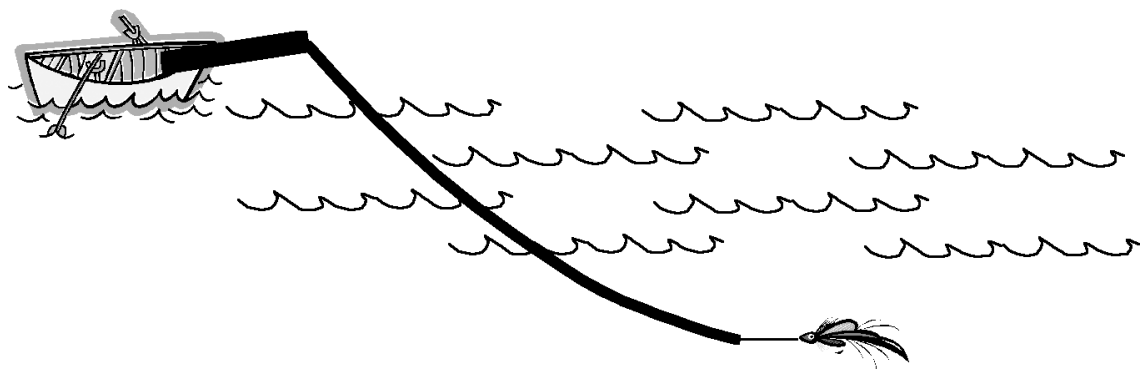

Figure 1 Commonly portrayed shape of a trolled heavy fishing line.

The correlation between lure depth and line payout depends on the shape that the line assumes while being towed. If one consults books and articles on trolling fishing flies, illustrations of the shape of the trolled line typically look like that shown in Fig. 1. We assume that the trolling line has a uniform diameter and density throughout its length, and the fly tends to have negligible impact on line shape during trolling. A curved line shape is normally portrayed because few, if any, have directly observed the actual line shape during trolling. A similar curved line shape is documented for a somewhat different trolling application that has been extensively studied: cable towing of submerged sonar arrays by ocean-going vessels.

A substantial body of research literature exists for towing sonar arrays by heavy cables, as discussed in several review articles (Paidoussis, 1987; Triantafyllou,
1991). For such applications, the cable shape is dictated by the cable properties, the hydrodynamic drag on the cable, and the properties of the towed cylinder containing the sonar array representing an end load. In the limiting case, where the towed body is very small or missing completely, theory predicts that the towed cable is straight rather than curved as it hangs in the water (Dowling, 1988a; Dowling, 1988b). The straight line shape, in the direction of towing motion, has been confirmed by experiment (Facchinetti et al., 2002). Most related studies employ the straight line shape as the baseline to predict outof-plane cable oscillations, which are immaterial to trolled fishing line prediction. Nonetheless, this same basic method of analysis applies and is employed here to develop a theory for trolled fishing lines.

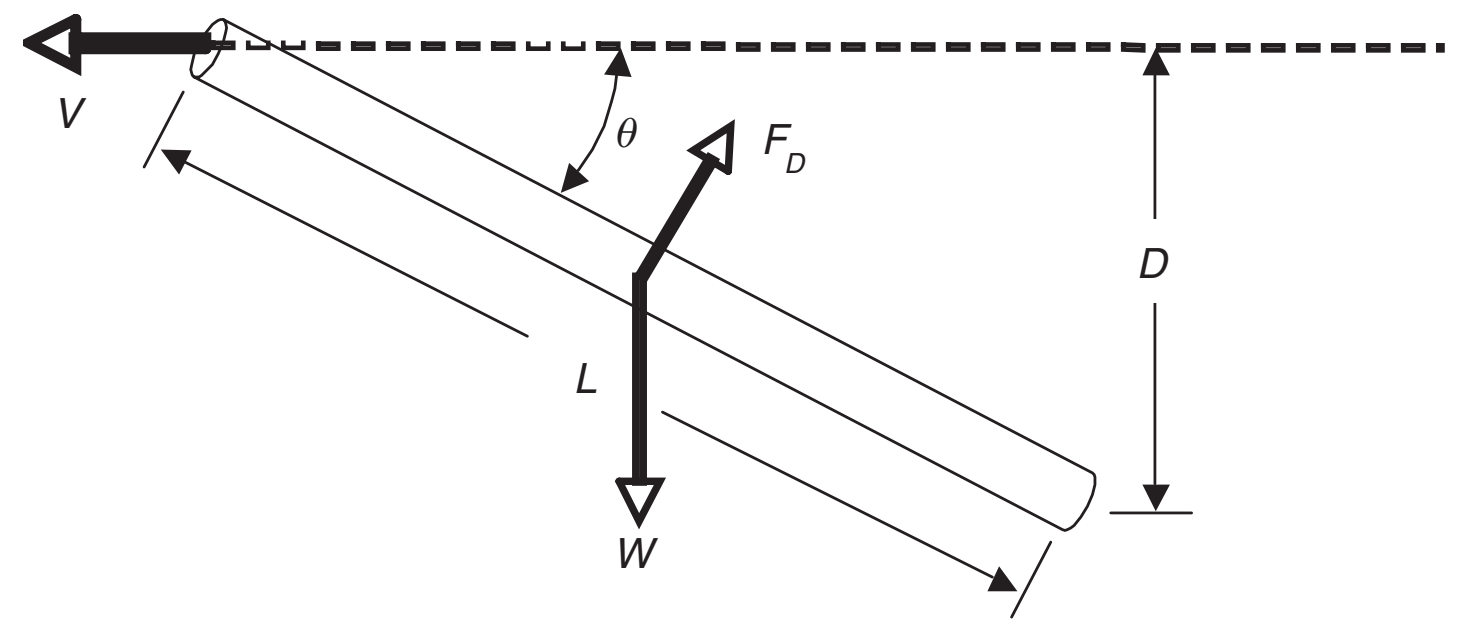

Figure 2 Model of trolled fishing line. 


\section{Theory}

A trolled fishing line can be modelled as a uniform, flexible cylinder of diameter $d$ and length $L$ suspended in quiescent water. The trolled end, which is attached to the fishing rod and boat, is drawn at a constant velocity $V$. With nothing attached to the terminal end of the line, the line will hang straight at a critical angle $\theta$ (Dowling, 1988b) to the water surface, as illustrated in Fig. 2.

Since the line's position is not changing, laws of statics can be used to balance the forces acting on the line segment. For low Reynolds number flow, the drag force $F_{D}$ on the oblique line segment can be expressed as the drag force normal to the line segment, yielding

$$
F_{D}=C_{D} A_{P} \frac{1}{2} \rho V_{N}^{2}
$$

where $C_{D}$ is the drag coefficient, $A_{P}$ is the projected area $\left(A_{P}=d L\right), \rho$ is the water density and $V_{N}$ is the velocity component normal to the cylinder, which in this case $V_{N}=V \sin \theta$. Accounting for buoyancy acting on the line with specific gravity $\sigma$ with gravitational constant $g$, the force balance yields an equation for the angle of inclination of the line as

$(\sigma-1)\left(\frac{\pi}{4} d^{2} L\right)(\rho g) \frac{L}{2} \cos \theta=\frac{L}{2} C_{D}(d L)\left[\frac{1}{2} \rho(V \sin \theta)^{2}\right]$

which simplifies to

$$
(\sigma-1)\left(\frac{d g}{2 V^{2}}\right) \cos \theta=\frac{C_{D}}{\pi} \sin ^{2} \theta
$$

Solving eqn. 3 for line angle $\theta$ then yields the depth of the line tip as

$$
D=\mathrm{L} \sin \theta
$$

Eqn. 3 is equivalent to that derived by Dowling (1988b) for a heavier-than-water cable with no end drag from a neutrally buoyant tool being towed. Dowling uses a constant value of $C_{D}=1.2$ for Reynolds numbers $\left(\operatorname{Re}=V_{N} d / v\right)$ in the neighborhood of $3 \times 10^{5}$, but for fishing lines both the towing speed $V$ and the line diameter $d$ are substantially lower, yielding Reynolds numbers more typically in the range of 20-250 for water with kinematic viscosity $v$. Published data for $C_{D}$ in this range
(Tritton, 1959; Wieselberger, 1921) can be curve-fit using the formula (White, 1974)

$$
C_{D}=1.0+\frac{10.0}{\mathrm{Re}^{2 / 3}} .
$$

Defining the dimensionless group $N$ as

$$
N=\frac{\pi}{2} \cdot \frac{g(\sigma-1) d}{C_{D} V^{2}}
$$

allows eqn. 3 to be rewritten as

$$
\cos ^{2} \theta+N \cos \theta-1=0
$$

which can be solved explicitly for line inclination angle $\theta$.

Two important observations may be drawn from this simple model:

1 The steady state trolled angle of inclination $\theta$ depends on specific gravity $\sigma$, line diameter $d$ and towing speed $V$, but does not depend on line length $L$. Furthermore, $\theta$ increases monotonically with the dimensionless group $N$ (see eqn. 6). Since this variable group $N$ alone determines $\theta$, it will hereafter be referred to as the 'trolled line parameter'.

2 For a given value of the trolled line parameter $N$, the depth $D$ of the line tip varies linearly with line length $L$.

\section{Experimental study}

An experimental study was conducted to examine whether the simple trolled line model could be used to predict accurately the depth of the line's free end. Specifically, there were two objectives:

1 to validate that depth $D$ varies linearly with line length $L$ for towed flexible line

2 to validate that line inclination angle $\theta$ varies with the trolled line parameter $N$.

The experimental methods employed were representative of actual fishing conditions, even though no hooks were employed nor fish disturbed by the tests. Fishing lines tested were selected from those available to the fishing community; hence they possessed the same range of flexibility, diameter, and specific gravity as lines commonly used for fishing. The physical 
properties of each line were measured in the laboratory. A precision micrometer was used to measure line diameter. A tape measure was used to measure (and mark) line length. Specific gravity was measured using the method of Havstaad (1979) requiring measurement of the weight of each line in air and while submersed in water. These properties are listed in Table 1.

Table 1 Heavy fishing line properties

\begin{tabular}{lcc}
\hline Fishing line & Specific gravity & Diameter $(\mathrm{mm})$ \\
\hline Lead core & 3.29 & 1.04 \\
Lead wire & 10.8 & 0.51 \\
Type 2 fly line & 1.42 & 1.01 \\
Type 6 fly line & 3.19 & 0.84 \\
Electrical wire & 1.33 & 5.59 \\
\hline
\end{tabular}

\section{Test 1 - Angle of towed line}

The first experiment measured the inclination angle $\theta$ for a fishing line towed at a known constant speed. A portable apparatus was constructed to measure the line angle relative to the water surface. The apparatus employed a protractor mounted to a bubble level and attached to the side of a boat so that it could be adjusted to maintain a horizontal orientation. The angle formed by the towed line could be readily measured.

All tests were performed on a cold, still, deep lake. Water temperature was measured at $4.5^{\circ} \mathrm{C}$, corresponding to a kinematic viscosity $v=1.54 \times 10^{-6} \mathrm{~m}^{2} \mathrm{~s}^{-1}$. The boat was propelled by an electric trolling motor which maintained constant boat speed and straight line direction, as determined by GPS data. For each test, a constant length of line, between 5 and 7 metres long, was employed. Typical trolling speeds between $0.8-4.3 \mathrm{~km} \mathrm{~h}^{-1}$ were achieved.

\section{Test 2 - Depth of towed line}

Separate tests are performed to determine if the depth of a towed fishing line varies linearly with line length, as predicted by eqn. 4 . The fishing line in this test was simulated by an electrical cable with the properties listed in Table 1. Three conductors through the cable were connected to a miniature strain gauge pressure transducer relating measured hydrostatic pressure to

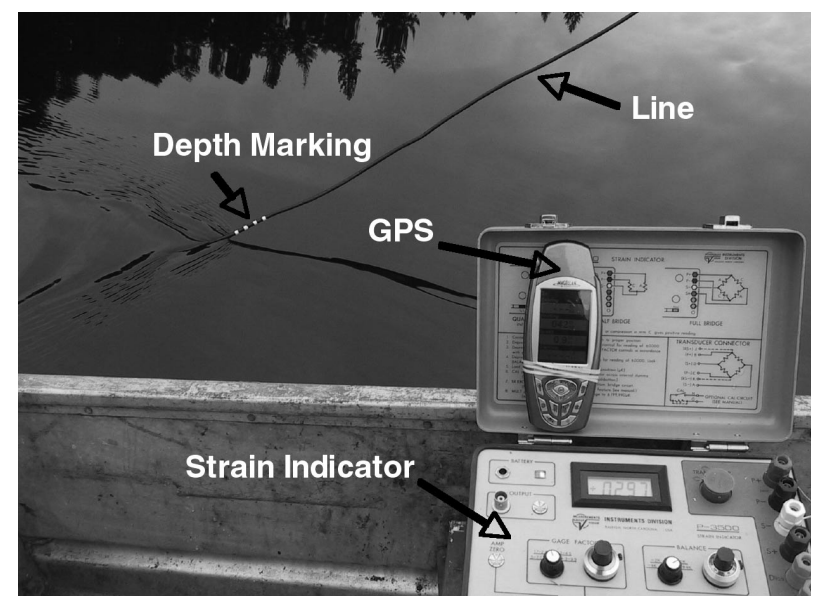

Figure 3 Measurement of depth of a trolled fishing line.

water depth. The diameter and thickness of the pressure transducer were $2.5 \mathrm{~mm}$ and $0.5 \mathrm{~mm}$, respectively, and its mass was less than $0.05 \mathrm{gm}$. When connected to the terminal end of the electric cable, minimal flow disturbances were added by the transducer so that the cable reacted as if there was no end load. A portable strain indicator was used to record the pressure signal as a function of depth.

The cable/transducer sank vertically by a heavy weight into the still lake to specific depths and the transducer output was recorded, supplying the calibration necessary, since the gauge also responded to depth-dependent temperature variation within the lake. The calibration proved to be linear. At a constant trolling speed, different line lengths were paid out and depth readings are recorded at steady state conditions. Boat speed was then changed and the procedure was repeated. Each run provided data on line tip depth versus line length. A photograph of the experimental set-up is shown in Fig. 3.

\section{Results}

The experimental results confirm that the model developed to predict the sinking depth of heavy fishing lines is accurate to within experimental uncertainty and can be employed for prediction of all heavy trolling lines.

The depth of the free end of a trolled line, as measured by a tip-mounted pressure transducer, verifies a linear relationship of depth to line length. 

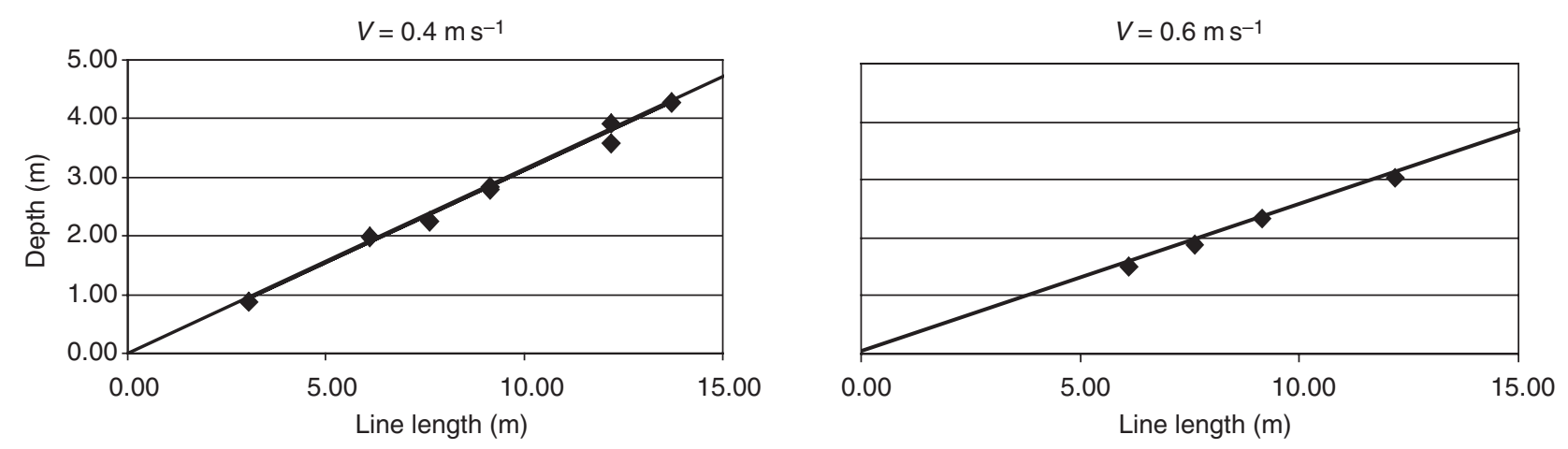

Figure 4 Experimental validation that line tip depth varies linearly with line length.

For a fishing line of known diameter and specific gravity, when towed at a constant speed, the tip depth depended only upon the line length as predicted by eqn. 4. Fig. 4 illustrates that the depth varies linearly with length for various trolling speeds.

The angle of inclination $\theta$ for a towed fishing line depends on the trolled line parameter $N$. Using measured values for specific gravity $\sigma$, line diameter $d$, towing speed $V$, and drag coefficient $C_{D}$ as predicted by eqn. 5, experimental results for the four lines listed in Table 1 are compared with theory in Fig. 5. As is observed from Fig. 5, the agreement is favourable between theory and experiment, and well within the experimental uncertainty for all measured values.

Based on these results, the experimental model can be used to develop tools for troll fishermen to allow them to regulate the depth of their fly or lure when using a heavy line. For example, manufacturers can

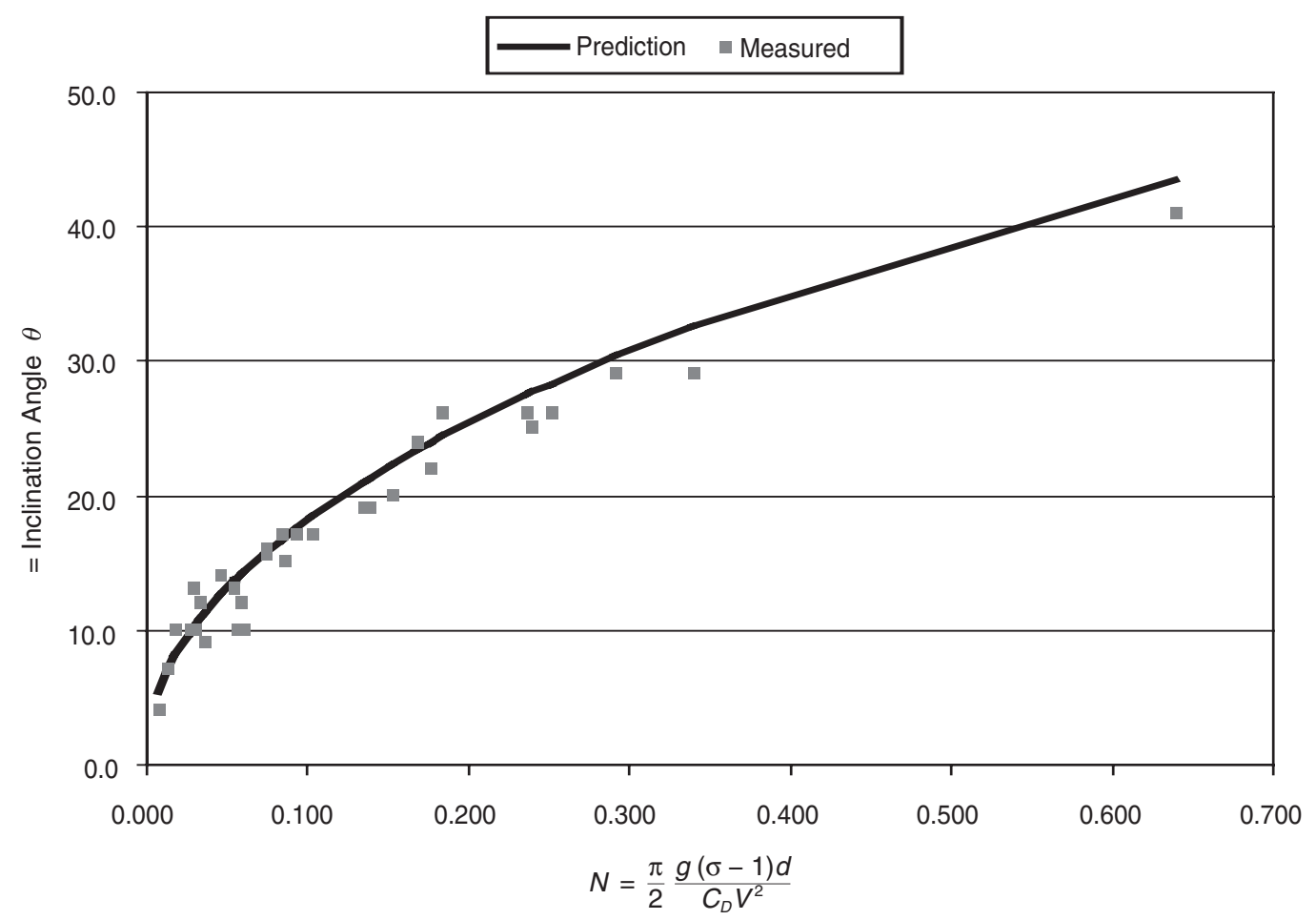

Figure 5 Comparison of experimental and theoretical towed line angle. 


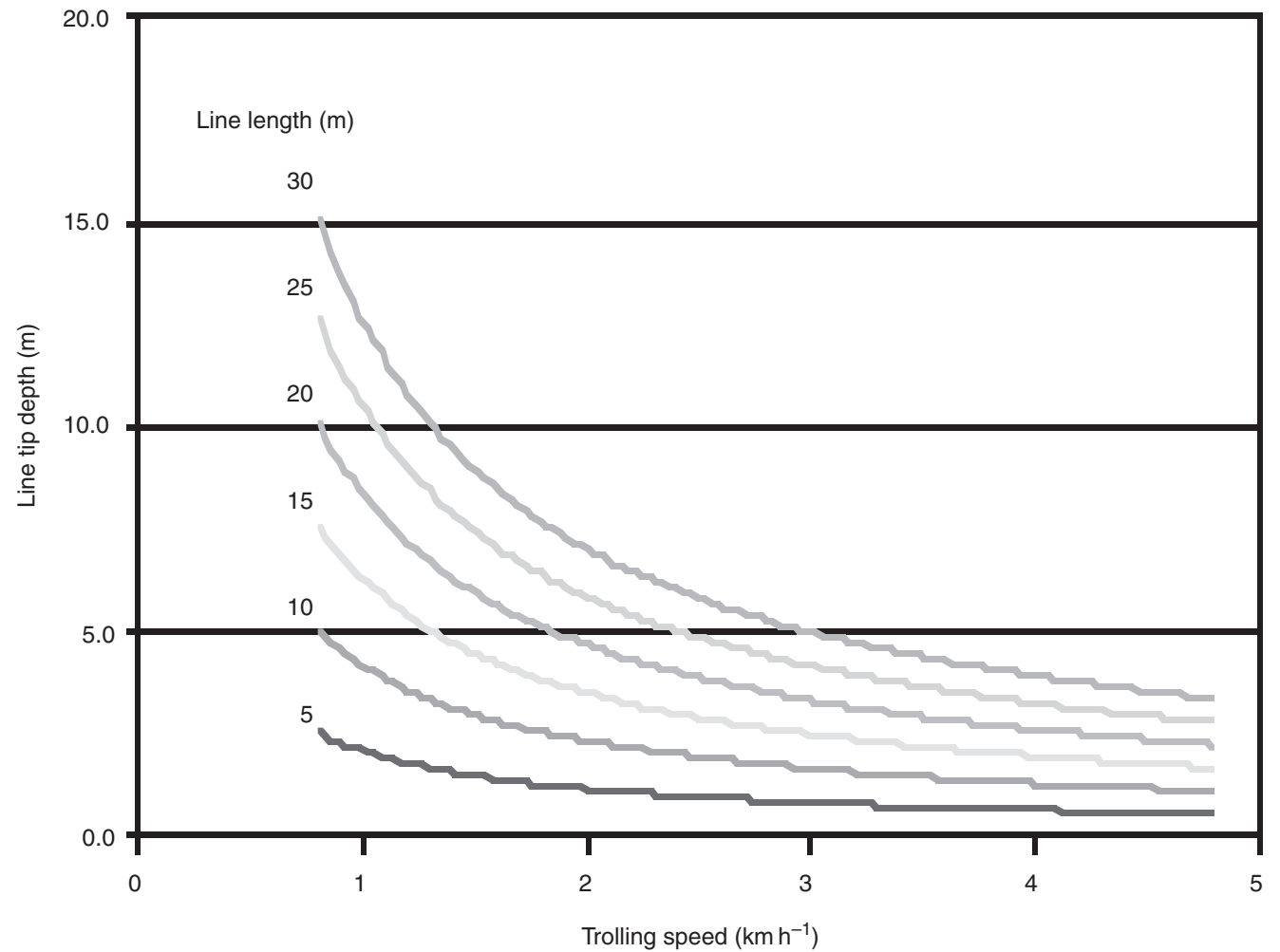

Figure 6 Line depth prediction chart for typical trolling line.

determine a line's specific gravity and diameter, and then supply a chart for the line allowing accurate prediction of depth for a known trolling speed and line length. Trolling lines would be marked to allow for appropriate line length to be paid out, while trolling speed would be available from on-board GPS data. Fig. 6 illustrates what such a chart might look like for a fly line with $\sigma=3.0$ and $d=0.84 \mathrm{~mm}$. As an example, if the fish were known to reside $5 \mathrm{~m}$ below the surface and this line were to be trolled at $1.0 \mathrm{~km} \mathrm{~h}^{-1}$, about $10 \mathrm{~m}$ of line would need to be paid out. On the other hand, if the boat speed were to be increased to $3.0 \mathrm{~km} \mathrm{~h}^{-1}$, then the line length would need to be increased to $30 \mathrm{~m}$ to maintain the lure $5 \mathrm{~m}$ below the surface. By adjusting line payout, the angler could be certain to maintain optimal lure depth and improve the odds of success.

\section{Conclusions}

Lure depth while troll fishing with heavy lines can be predicted and controlled to enhance fishing success:

- A simple model of line mechanics that balances hydrodynamic and body forces yields a prediction of trolled line depth for known line properties of specific gravity, diameter, and trolling speed.

- Experiments performed on different trolling lines towed at different speeds verifies that heavy, flexible fishing lines remain straight in the water with the inclination angle predicted by the model.

- As a service to customers, fishing line manufacturers could supply lines with length markings to facilitate accurate line payout, along with tables or charts that predict line depth as a function of trolling speed to control lure depth, optimising fishing success. 


\section{References}

Dowling, A.P. (1988a) The dynamics of towed flexible cylinders Part 1 . Neutrally buoyant elements. Fournal of Fluid Mechanics, 187, 507-532.

Dowling, A.P. (1988b) The dynamics of towed flexible cylinders Part 2. Negatively buoyant elements. Fournal of Fluid Mechanics, 187, 533-571.

Facchinetti, M.L., de Langre, E. \& Biolley, F. (2002) Experiments on vortex-induced traveling waves along a cable. Proceedings of the Third Conference on Bluff Body Wakes and Vortex-Induced Vibrations (BBVIV3). pp. 215-217. Port Douglas, Australia.

Havstad, J. (1979) Sink rate of sinking lines. The Flyfisher, Fall, 35-42.
Paidoussis, M.P. (1987) Flow induced instabilities of cylindrical structures. Applied Mechanics Review. Transactions of ASME, 40 (2), 163-175.

Triantafyllou, M.S. (1991) Dynamics of cables, towing cables, and mooring systems. Shock \& Vibration Digest, 23 (3), 3-8.

Tritton, D.J. (1959) Experiments on the flow past a circular cylinder at low Reynolds numbers. Fournal of Fluid Mechanics, 6, 547-567.

White, F.M. (1974) Viscous Fluid Flow. McGraw-Hill, New York, USA.

Wieselsberger, C. (1921) Neure festellungen über die gesetze des flüssigkeit- und luftwiderstands. Physikalische Zeitschrift, 22, 321-328. 
10.3.4 Sports F84 15/1/08 16:47 Page 172 